\title{
Requirements for Enhanced Transgene Expression by Untranslated Sequences from the Human Cytomegalovirus Immediate-Early Gene
}

\author{
Robert D. Simari, ${ }^{1}$ Zhi-Yong Yang, ${ }^{2}$ Xu Ling, ${ }^{2}$ \\ Dominique Stephan, ${ }^{2}$ Neil D. Perkins, ${ }^{3}$ Gary J. Nabel, ${ }^{3}$ and \\ Elizabeth G. Nabel ${ }^{2}$ \\ ${ }^{1}$ Division of Cardiovascular Diseases and Biochemistry and Molecular \\ Biology, Molecular Medicine Program, Mayo Clinic and Foundation, \\ Rochester, Minnesota, U.S.A. \\ ${ }^{2}$ Departments of Internal Medicine and Physiology, University of \\ Michigan, Ann Arbor, Michigan, U.S.A. \\ ${ }^{3}$ Howard Hughes Medical Institute, University of Michigan, Ann \\ Arbor, Michigan, U.S.A.
}

Communicated by E. Nabel. Accepted September 21, 1998.

\begin{abstract}
Background: The cytomegalovirus immediate early (CMV IE) promoter has been widely used for heterologous expression. Further enhancements of gene expression from this potent promoter may allow for the development of improved gene transfer strategies. We aimed to determine whether inclusion of the first exon $\left(5^{\prime}\right.$ untranslated) and first intron of the CMV IE gene would increase heterologous transgene expression in primary target cells and to determine the sequences required for any observed increases.

Materials and Methods: Comparisons of reporter gene expression were made following transient transfection of vascular smooth muscle cells (VSMCs) with plasmids containing the first exon and intron from the CMV IE gene or deletional mutations. Comparisons were also made using a heterologous promoter (RSV).

Results: Gene expression from the CMV IE promoter
\end{abstract}

\section{Introduction}

A goal of gene transfer is to modulate gene expression in host cells by the introduction of genes

Address correspondence and reprint requests to: Dr. Robert D. Simari, Mayo Clinic, 200 1st Street, SW, Rochester, MN 55904, U.S.A. Phone: (507) 284-3727; Fax: (507) 266-

4797; E-mail: simari.robert@mayo.edu was increased 5.7-fold in VSMC with the inclusion of the first exon and intron. Similar increases were seen with other target cells and from the heterologous RSV promoter. This increase was associated with an increase in steady-state mRNA. Deletion analyses demonstrated that the enhancement was dependent on the presence of the $5^{\prime}$ portion of the first exon while deletion of large segments within the intron was associated with similar levels of expression compared with the parental plasmid. Conclusions: Inclusion of the first exon and intron from the CMV IE gene increases expression from the CMV IE promoter. This enhancement is seen with the heterologous RSV promoter and is associated with an increase in steady-state mRNA. Deletion analyses suggest that this enhancement is associated with inclusion of sequences within the $5^{\prime}$ portion of the first exon and inclusion of an intron. encoding native or foreign proteins. Although viral vectors have several actual and theoretic advantages over nonviral vectors, limitations related to their immunogenicity and toxicity remain. Nonviral gene transfer has several important features, including its overall safety and lack of toxicity, but has been limited by the duration 
and extent of recombinant gene expression in primary target cells $(1,2)$. The use of plasmid DNA to express transgenes from skeletal muscle for either systemic expression or immunization in vivo has been an important demonstration of its potential (3). Strategies to improve the efficacy of nonviral vectors include the development of new cationic liposomes and the development of enhanced expression plasmids. Novel cationic liposomes, including GAP DLRIE/DOPE $(4,5)$ have increased in vivo gene expression. Enhancement of gene expression from plasmids might be obtained by developing plasmids that are capable of episomal replication (6), by creating tissue specificity, or by altering the regulation of gene expression from the plasmid. The cytomegalovirus immediate early (CMV IE) promoter has been widely used as a potent constitutive viral promoter in expression plasmids for nonviral transfection $(7,8)$. Addition of intronic sequences to expression plasmids has been shown to increase transgene expression in transient transfections in some transformed cell lines $(9,10)$ and in the development of transgenic animals $(11,12)$. It has been suggested that the $5^{\prime}$ untranslated portion (first exon) and first intron of the CMV IE gene may be involved in the regulation of gene expression $(9,10)$ from the CMV IE promoter. However, the extent and mechanism of regulation may vary with target cell and the transgene to be expressed. To increase gene expression following nonviral delivery of DNA, we investigated whether addition of untranslated sequences from the CMV IE gene would increase transgene expression in vascular smooth muscle cells (VSMCs), a target for vascular gene transfer, and to determine the sequences required for this effect.

\section{Materials and Methods}

\section{Plasmid Development}

The eukaryotic expression plasmid pCMVintCAT was obtained from Vical Inc. (San Diego, CA); it expresses the chloramphenicol acetyl transferase gene under the control of the CMV IE enhancer/ promoter (Fig. 1). This plasmid also contains the first exon ( $5^{\prime}$ untranslated) and first intron from the CMV IE gene and a bovine growth hormone polyadenylation sequence. The plasmid pCMVCAT contains the same backbone as pCMVintCAT with the entire $5^{\prime}$ untranslated and first intron sequences deleted. The plasmid pCMVint(SacII)CAT contains $69 \mathrm{bp}$ of the $5^{\prime}$ untranslated

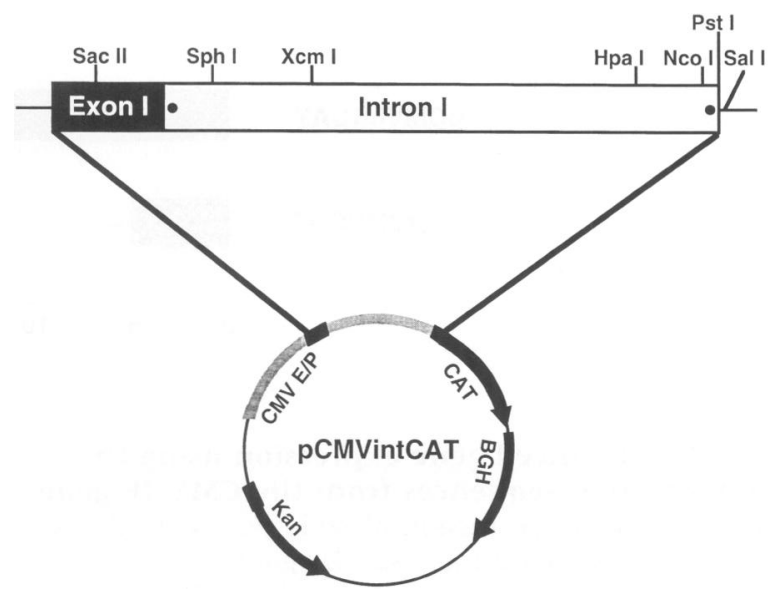

Fig. 1. A map of the plasmid pCMVintCAT including restriction sites used to develop deletional mutations.

sequences with deletion of the intron. The plasmid pCMVint(S/N)CAT contains an internal intron deletion from the $S p h \mathrm{I}$ restriction site to the $N c o$ I restriction site and contains $72 \mathrm{bp}$ of the intron. The plasmid pCMVint $(\mathrm{X} / \mathrm{N}) \mathrm{CAT}$ contains an internal intron deletion from the $X \mathrm{~cm} I$ restriction site to the $\mathrm{NcoI}$ restriction site and contains $179 \mathrm{bp}$ of the intron. The plasmid pCMVint(S/ $\mathrm{H}) \mathrm{CAT}$ contains an internal intron deletion from the SphI restriction site to the $\mathrm{HpaI}$ restriction site and contains $165 \mathrm{bp}$ of the intron. The plasmid pCMVint $(\mathrm{X} / \mathrm{H}) \mathrm{CAT}$ contains an internal intron deletion from the $\mathrm{XcmI}$ restriction site to the $\mathrm{HpaI}$ restriction site and contains $273 \mathrm{bp}$ of the intron.

The plasmid pRSVCAT expresses CAT under the control of the RSV promoter with the bovine growth hormone polyadenylation signal. The plasmid pRSVintCAT contains the $5^{\prime}$ untranslated and first intron from the CMV IE gene upstream of the CAT cassette. The plasmid pRSVint(SacII)CAT contains 69 bp of the $5^{\prime}$ untranslated sequences with deletion of the intron. All plasmids were purified by double cesium chloride banding.

\section{Transfection Protocol}

Porcine aortic vascular smooth muscle cells were obtained by standard explantation methods and used for transfection studies within the first six passages (13). Cells were grown to $30-40 \%$ confluence in 6- or 24-well plates (for duration of expression studies) in Medium 199 (Gibco/BRL) containing $10 \%$ fetal bovine serum. Cells were washed twice with phosphate-buffered saline 


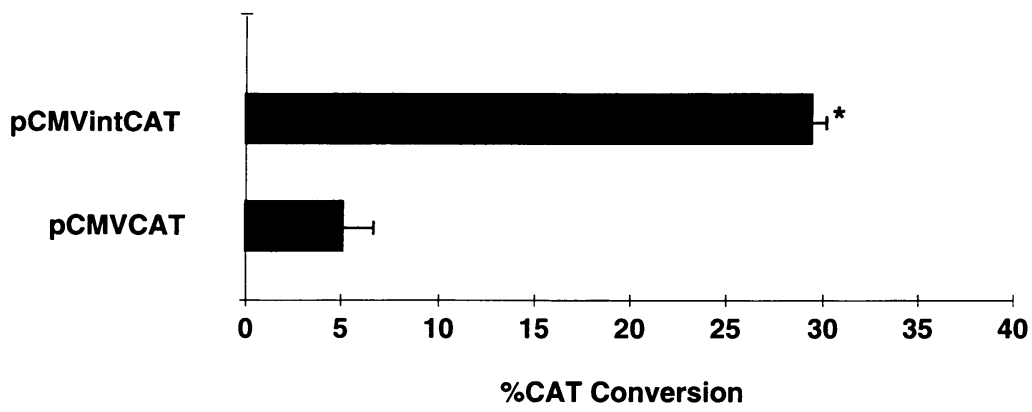

Fig. 2. Enhanced gene expression using the untranslated sequences from the CMV IE gene. The eukaryotic expression plasmid pCMVintCAT was obtained from Vical Inc. (San Diego, CA) and expresses the chloramphenicol acetyl transferase gene under the control of the CMV IE enhancer/promoter. This plasmid also contains the $5^{\prime}$ untranslated and first intron from the CMV IE gene and a bovine growth hormone polyadenylation sequence. The plasmid pCMVCAT contains the same backbone as pCMVintCAT with the entire $5^{\prime}$ untranslated and first intron sequences deleted ( $B s m \mathrm{BI}$ to $E c o \mathrm{RV})$. Por- cine aortic vascular smooth muscle cells were obtained by standard explantation methods and used for transfection studies within the first six passages. Cells were grown to $30-40 \%$ confluence in 6-well plates in Medium 199 (Gibco/BRL) containing 10\% fetal bovine serum and transfected with $5 \mu \mathrm{g}$ of plasmid DNA and $10 \mu \mathrm{g}$ of a cationic liposome, DMRIE/ DOPE (Vical) (23). Transfections were performed in triplicate. Detection of CAT activity was performed as previously described (14). ${ }^{*} p=0.002$ using unpaired two-tailed Student's $t$-test.
(PBS) prior to addition of DNA liposomes. DNA liposomes were prepared by adding $5 \mu \mathrm{g}$ of plasmid DNA to $0.5 \mathrm{ml}$ of Opti-MEM (Gibco/BRL). A cationic lipid, DMRIE/DOPE (Vical), was prepared by adding $10 \mu \mathrm{g}$ of DMRIE/DOPE to $0.5 \mathrm{ml}$ of Opti-MEM (Gibco/BRL). Prior to transfection, the DNA solution was added to the liposomes and gently mixed. The transfection solution was then added to each well and plates were kept at $37^{\circ} \mathrm{C}$ for $2 \mathrm{hr}$. Following transfection, the cells were washed twice with PBS, Medium 199 was added and the cells were kept at $37^{\circ} \mathrm{C}$. The cells were harvested at $1,5,9$, or 20 days following transfection. Transfections were performed in triplicate. Transfections were performed in an identical fashion for 293 cells (American Type Culture Collection, Rockville, MD).

\section{CAT Assay}

To determine the extent of CAT expression within each sample, protein was extracted by freezing and thawing three times. Protein was quantified by means of a calorimetric assay (BioRad, Hercules, CA). The amount of protein used in each assay was the same among each group of comparisons and ranged from $0.2 \mu \mathrm{g}$ to $20 \mu \mathrm{g}$. CAT activity was determined during a 20- to 30-min incubation as previously described (14) and expressed as \% acetylation of $\left[\mathrm{C}^{14}\right]$ chloramphenicol.

\section{Northern Blot Analysis}

293 cells were grown in 10-cm dishes and transfected with either pCMVCAT or pCMVintCAT as above. Nontransfected 293 cells were used as controls. Cells were harvested $24 \mathrm{hr}$ after transfection. Total RNA was isolated using RNAZOL B (Tel-Test, Inc., Friendswood, TX). Northern blot analysis was performed using standard techniques (Northern Max, Ambion Inc, Austin, TX). Briefly, $20 \mu \mathrm{g}$ of total RNA was electrophoresed on a $1 \%$ denaturing formamide gel and transferred to a nitrocellulose membrane using the Turbo Blot (Schleicher \& Schuell; Keene, NH) method of capillary transfer. Membranes were cross-linked, then prehybridized for $2 \mathrm{hr}$ at $42^{\circ} \mathrm{C}$, followed by hybridization overnight at $42^{\circ} \mathrm{C}$ with a random prime-labeled $\left(\alpha-{ }^{32} \mathrm{P}\right.$-dCTP $)$ cDNA probe (Prime It II Random Prime Labeling Kit, Stratagene, La Jolla, CA). CAT mRNA bands were subsequently visualized on X-ray film. To control for variations in RNA loading, membranes were stripped of previous labeled CAT probe by boiling in $1 \%$ SDS solution and rehybridized with a cDNA probe to human GAPDH (Ambion).

\section{Statistics}

Results are expressed as a mean and a standard error of the mean (S.E.M.). Each transfection was treated as an independent observation. 


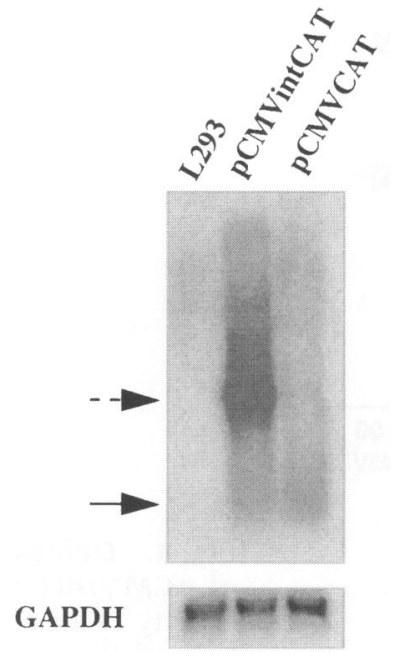

Fig. 3. Addition of $5^{\prime}$ sequences from CMV IE gene is associated with an increase in steadystate mRNA. Northern analysis of RNA from 293 cells transfected with pCMVintCAT (lane 2) or pCMVCAT (lane 3) with nontranfected 293 cells (lane 1). The upper band (1.6 kb) (dotted arrow) is the predominant band from pCMVintCAT transfections compared with the expected $0.7 \mathrm{~kb}$ band (solid arrow) also seen in the pCMVCAT transfections consistent with CAT mRNA.

Comparisons were made between samples when identical amounts of protein and incubation times were used and thin-layer chromatography was performed simultaneously. The means of groups were compared by an unpaired twotailed Student's $t$-test or by analysis of variance (ANOVA) with Dunnet $t$-test. Statistical significance was assumed if a null hypothesis could be rejected at the 0.05 level.

\section{Results}

To examine the effect of the entire CMV IE untranslated sequences on transgene expression in VSMCs, comparisons were made between pCMVintCAT and pCMVCAT. Addition of the complete untranslated sequence from the CMV IE gene downstream of the CMV enhancer/promoter resulted in a 5.7-fold increase in gene expression $(p=0.002)$ (Fig. 2). A similar enhancement was seen in other cell types, including peripheral blood lymphocytes and human embryonic kidney (293) cells (data not shown).

To determine the mechanism for this enhancement, Northern blot analyses were performed. Transfections were performed and RNA was isolated $24 \mathrm{hr}$ following transfection. These studies demonstrate an increase in steady-state RNA associated with the inclusion of the $5^{\prime}$ untranslated sequences. However, this increase is primarily due to the presence of a higher-molecular-weight (MW) band from the cells transfected with pCMVintCAT (Fig. 3). The higher-MW $(1.6 \mathrm{~kb})$ form from the pCMVintCAT transfected cells was the dominant band compared to the $0.7 \mathrm{~kb}$ band expected for CAT seen with both transfections. Thus, the increase in transgene expression with inclusion of the $5^{\prime}$ untranslated sequences was associated with a dominant higher-MW form of RNA than that expected for CAT mRNA.

To determine the requirements for the $5^{\prime}$ untranslated exon and the first intron, a series of deletional mutations was created using inherent restriction sites (Fig. 4A). Large internal deletions from within the intron failed to significantly decrease gene expression (65-101\%), although the larger deletions were associated with a trend toward lower gene expression. Deletion of the entire intron and the $3^{\prime}$ portion of the first exon [pCMVint(Sac II)CAT] decreased gene expression by $56 \%(p=0.01)$. This deletion spares the sequences from the $5^{\prime}$ untranslated sequences previously identified as a positive regulatory domain (15).

To determine the effects of these sequences on the duration of gene expression following transfection, transfected VSMCs were maintained without splitting for up to 20 days. The pattern of decline in gene expression over time was similar to the comparison at 1 day; internal intron deletions did not affect the extent of gene expression, while deletion of the entire intron decreased gene expression (Fig. 4B). The degree of expression at any point appeared to be more dependent on the overall initial degree of expression rather than the rate of the decline in expression over time.

To determine the effects of adding the $5^{\prime}$ untranslated sequences downstream of a promoter with lower basal activity, a series of similar constructs were made with the RSV promoter. Addition of the entire untranslated sequences resulted in increased expression in VSMCs by 10.3-fold (Fig. 5) $(p=0.02)$. The addition of the first $69 \mathrm{bp}$ of the $5^{\prime}$ untranslated sequence from the CMV IE gene increased gene expression to a nonsignificant degree in VSMCs by 1.83 -fold $(p=\mathrm{NS})$. Therefore, the enhanced expression with addition of untranslated sequences from the CMV IE gene is not specific for expression from 

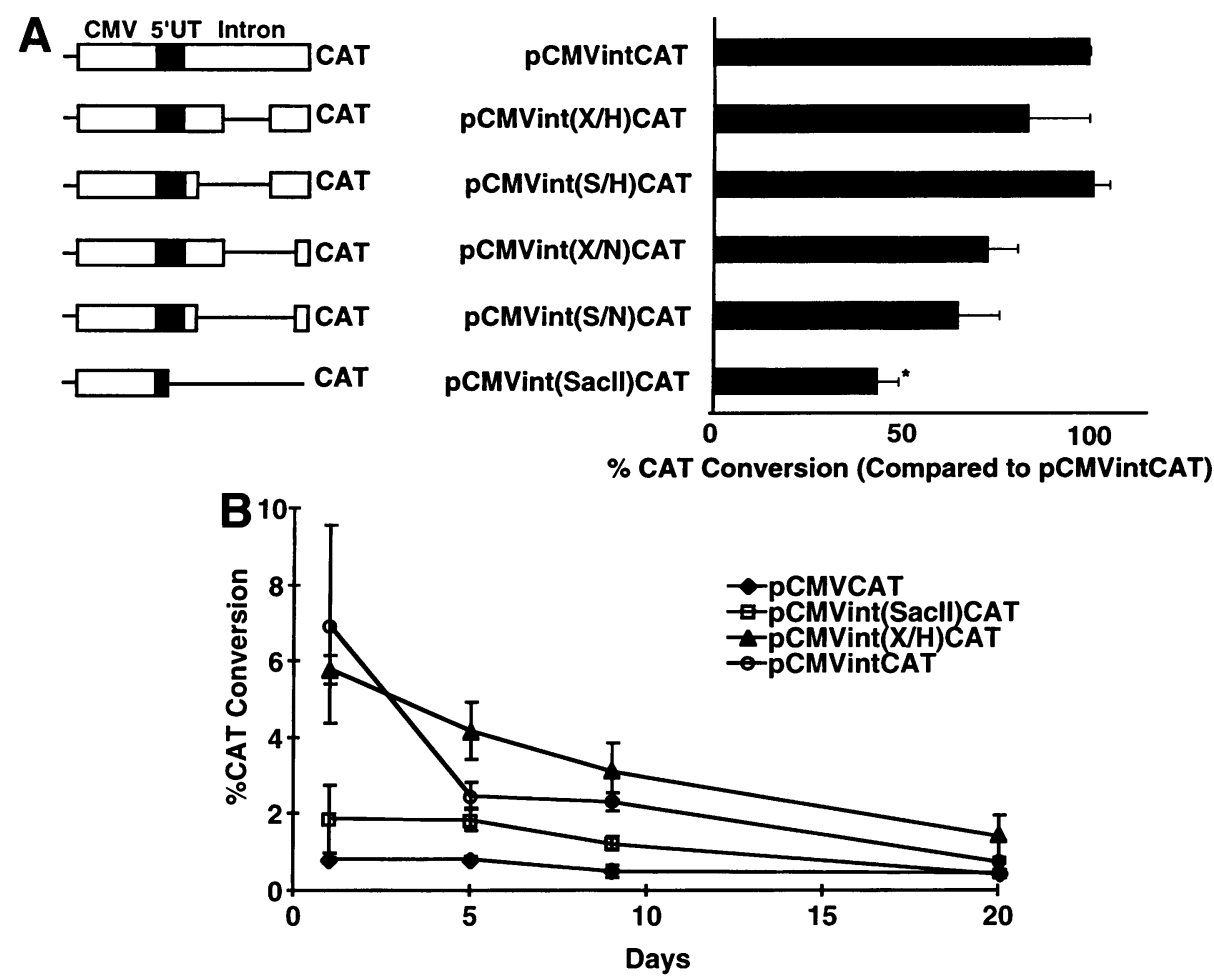

Fig. 4. Deletion analysis of pCMVintCAT. (A) CAT activity $24 \mathrm{hr}$ after transfection relative to $\mathrm{pCMVintCAT}$. ${ }^{*} p=0.01$ compared to $\mathrm{pCM}$ VintCAT by analysis of variance (ANOVA) with Scheffe's F-test. (B) Time course of transgene expression from plasmids containing untranslated sequences from the CMV IE gene. See text for description of plasmids.

the CMV IE promoter but is present with a heterologous viral promoter. The degree of increase with these sequences is greater than with the homologous promoter perhaps because the CMV promoter/enhancer is 100 -fold more active than the RSV promoter in this system (data not shown).

\section{Discussion}

Gene transfer studies may provide important insights into the pathophysiology and potential treatments of human disease. A limitation of gene transfer has been the difficulty in obtaining high levels of gene expression using nonviral methods of transfer. Vascular smooth muscle cells are potential targets for the treatment of vasculoproliferative diseases, such as restenosis and atherosclerosis. Although virally mediated gene transfer has proven effective in VSMCs, there are several advantages to nonviral approaches, including the lack of toxicity and simplicity. The safety of DNA liposomes and DNA alone have now been documented in several animal toxicity and human trials $(1,15-20)$.

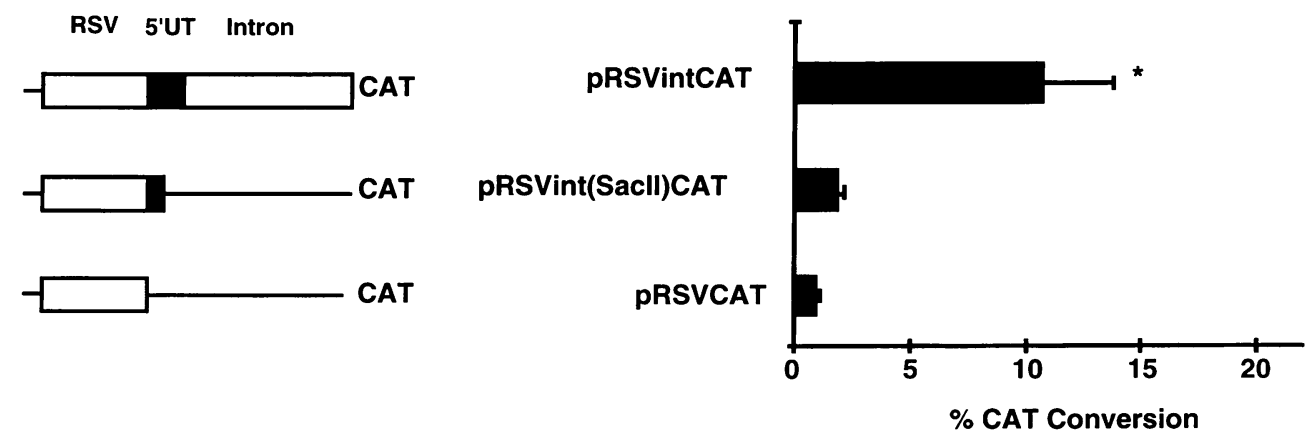

Fig. 5. Comparison of transgene expression from plasmids containing RSV promoter and untranslated sequences from CMV IE gene. See above for description of plasmids. ${ }^{*} p<0.05$ compared to pRSVCAT by analysis of variance (ANOVA) with Scheffe's F-test. 
In this report, gene expression using nonviral methods was enhanced by the addition of untranslated sequences from the CMV IE gene. Gene expression from the CMV IE promoter was increased 6-fold in target cells. This enhancement with these untranslated sequences is associated with a dominant higher-MW form of RNA than that expected for CAT. A previous study by Chapman et al. also noted this higher-molecularweight form of RNA associated with this intron, but they attributed this form to read-through of the weak SV40 polyadenylation sequence that was used in their study (9). Our study differs from this study in that our plasmids used the bovine growth hormone polyadenylation sequence and comparisons to intronless plasmids were performed. It is the presence of this higher-MW form that differentiates between the results from the two constructs. A possible explanation for this higher-MW form of RNA might be that it is unspliced and that its presence is associated with increased protein expression in these steady-state studies.

Deletion analyses demonstrated that the enhancement was dependent upon the presence of the $5^{\prime}$ untranslated exon and the presence of an intron. Deletion of large segments within the intron was associated with similar levels of expression compared with the parental plasmid. The greater degree of expression was seen throughout the time course of in vitro expression, but was not associated with a different time course of extinction. Expression from the weaker heterologous RSV promoter was increased 10fold with inclusion of these nontranslated sequences. Thus, the enhancement observed with inclusion of the sequences is not limited to the homologous promoter and is dependent on inclusion of sequences within the first exon and at least a minimal intron.

Intronic sequences may increase gene expression from plasmids by either stabilizing mRNA by splicing or polyadenylation (21) or by acting as an enhancer (22), and they have been used to enhance transgene expression in transgenic animals $(11,12)$. Some functional analyses of the 5' untranslated and intron from CMV IE gene have been reported previously. A regulatory domain within the first exon was identified by Ghazal and Nelson and was shown to enhance the number of functional initiation complexes by binding two discrete cellular proteins, named leader transcription factors $A$ and $B$ (LTFA and LTFB) (10). A separate analysis of the entire untranslated sequence demonstrated a variable effect on expression associated with an NFl site within the first intron (9). This variability appeared to be due to the specific transgene expressed.

The current studies were designed to determine whether inclusion of these untranslated sequences would enhance expression in target cells for gene transfer. In these studies, the significance of the initial portion of first exon is demonstrated by a 3-fold increase in gene expression. This increase may be due to the presence of the sequence previously shown to bind LTFA and LTFB. The additional increase in expression seen with the intron does not appear to be related to the NFl site as previously described. This site was deleted from all of the internal intron deletions without a significant decrease in gene expression. However, the presence of a minimal intron is associated with increased gene expression, suggesting either a transcriptional or post-transcriptional mechanism, such as mRNA stability as shown in previous studies. Taken together, these findings suggest that the inclusion of the first exon and intron from the CMV IE gene increases gene expression in transient transfections in VSMCs. This increase is seen in the presence of heterologous promoters and is dependent on the presence of the untranslated exon and a minimal intron. These findings might be used to develop potent gene delivery strategies that could achieve biological effects and avoid the potential toxicity of viral strategies.

\section{Acknowledgments}

We gratefully acknowledge T. Peterson and C. Mueske for technical expertise and M. Craft for manuscript preparation. This study was supported by a National Institutes of Health grant (HL03473), a grant-in-aid from the Minnesota affiliate of the American Heart Association, the Miami Heart Research Institute, and the Bruce and Ruth Rappaport Program in Vascular Biology.

\section{References}

1. Nabel E, Plautz G, Nabel G. (1990) Site-specific gene expression in vivo by direct gene transfer into the arterial wall. Science 249: 1285-1288.

2. Nabel E, Plautz G, Nabel G. (1992) Transduction of a foreign histocompatibility gene into the arterial wall. Proc. Natl. Acad. Sci. U.S.A. 89: 5157-5161.

3. Tripathy S, Svensson E, Black H, et al. (1996) Long-term expression of erythropoietin in the sys- 
temic circulation of mice after intramuscular injection of a plasmid DNA vector. Proc. Natl. Acad. Sci. U.S.A. 93: 10876-10880.

4. Wheeler CJ, Felgner PL, Tsai YJ, et al. (1996) A novel cationic lipid greatly enhances plasmid DNA delivery and expression in mouse lung. Proc. Natl. Acad. Sci. U.S.A. 93: 11454-11459.

5. Stephan DS, Yang ZY, San H, et al. (1996) A new cationic liposome DNA complex enhances the efficiency of arterial gene transfer in vivo. Hum. Gene Ther. 7: 1803-1812.

6. Cooper MJ, Miron S. (1993) Efficient episomal expression vector for human transitional carcinoma cells. Hum. Gene Ther. 4: 557-566.

7. Schmidt EV, Christoph G, Zeller R, Leder P. (1990) The cytomegalovirus enhancer: a pan-active control element in transgenic mice. Mol. Cell Biol. 10: 4406-4411.

8. Ghazal P, Lubon H, Fleckenstein B, Hennighausen L. (1987) Binding of transcription factors and creation of a large nucleoprotein complex on the human cytomegalovirus enhancer. Proc. Natl. Acad. Sci. U.S.A. 84: 3658-3662.

9. Chapman BS, Thayer RM, Vincent KA, Haigwood NL. (1991) Effect of intron A from human cytomegalovirus (Towne) immediate-early gene on heterologous expression in mammalian cells. $\mathrm{Nucl}$. Acids Res. 19: 3979-3986.

10. Ghazal P, Nelson JA. (1991) Enhancement of RNA polymerase II initiation complexes by a novel DNA control domain downstream from the cap site of the cytomegalovirus major immediateearly promoter. J. Virol. 65: 2299-2307.

11. Choi T, Huang M, Gorman C, Jaenisch R. (1991) A generic intron increases gene expression in transgenic mice. Mol. Cell Biol. 11: 3070-3074.

12. Palmiter RD, Sandgren EP, Avarbock MR, Allen DD, Brinster RL. (1991) Heterologous introns can enhance expression of transgenes in mice. Proc. Natl. Acad. Sci. U.S.A. 88: 478-482.

13. Simari R, San H, Rekhter M, et al. (1996) Regulation of cellular proliferation and intimal forma- tion following balloon injury in atherosclerotic rabbit arteries. J. Clin. Invest. 98: 225-235.

14. Gorman CM, Moffat LF, Howard BH. (1982) Recombinant genomes which express chloramphenicol acetyltransferase in mammalian cells. Mol. Cell Biol. 2: 1044-1051.

15. Chapman G, Lim C, Gammon R, et al. (1992) Gene transfer into coronary arteries of intact animals with a percutaneous balloon catheter. Circ. Res. 71: 27-33.

16. Leclerc G, Gal D, Takeshita S, Nikol S, Weir L, Isner J. (1992) Percutaneous arterial gene transfer in a rabbit model. Efficiency in normal and balloon-dilated atherosclerotic arteries. J. Clin. Invest. 90: $936-944$.

17. Muller D, Gordon D, San H, et al. (1994) Catheter-mediated pulmonary vascular gene transfer and expression. Circ. Res. 75: 1039-1049.

18. Nabel GJ, Nabel EG, Yang Z, et al. (1993) Direct gene transfer with DNA-liposome complexes in melanoma: expression, biologic activity, and lack of toxicity in humans. Proc. Natl. Acad. Sci. U.S.A. 90: 11307-11311.

19. San H, Yang Z, Pompili V, et al. (1993) Safety and toxicity of a novel cationic lipid formulation of human gene therapy. Hum. Gene Ther. 4: 781-788.

20. Takeshita S, Gal D, Leclerc G, et al. (1994) Increased gene expression after liposome-mediated arterial gene transfer associated with intimal smooth muscle cell proliferation. J. Clin. Invest. 93: 652-661.

21. Niwa M, Rose SD, Berget SM. (1990) In vitro polyadenylation is stimulated by the presence of an upstream intron. Genes Dev. 4: 1552-1559.

22. Buchman AR, Berg P. (1988) Comparison of intron-dependent and intron-independent gene expression. Mol. Cell Biol. 8: 4395-4405.

23. Stewart M, Plautz G, Buono L, et al. (1992) Gene transfer in vivo with DNA-liposome complexes: safety and acute toxicity in mice. Hum. Gene Ther. 3: 267-275. 